\title{
Domain Decomposition and Model Reduction of Systems with Local Nonlinearities
}

\author{
Kai Sun, Roland Glowinski, Matthias Heinkenschloss, and Danny C. Sorensen
}

\begin{abstract}
The goal of this paper is to combine balanced truncation model reduction and domain decomposition to derive reduced order models with guaranteed error bounds for systems of discretized partial differential equations (PDEs) with a spatially localized nonlinearities. Domain decomposition techniques are used to divide the problem into linear subproblems and small nonlinear subproblems. Balanced truncation is applied to the linear subproblems with inputs and outputs determined by the original in- and outputs as well as the interface conditions between the subproblems. The potential of this approach is demonstrated for a model problem.
\end{abstract}

\section{Introduction}

Model reduction seeks to replace a large-scale system of differential equations by a system of substantially lower dimension that has nearly the same response characteristics. This paper is concerned with model reduction of systems of discretized partial differential equations (PDEs) with spatially localized nonlinearities. In particular, we are interested in constructing reduced order models for which the error between the input-to-output map of the original system and the input-to-output map of the reduced order model can be controlled.

Balanced truncation is a particular model reduction technique due to [16], which for linear time invariant systems leads to reduced order models which approximate the original input-to-output map with a user controlled error [1, 6]. Although extensions of balanced truncation to nonlinear systems have been proposed, see, e.g.,

Kai Sun, Matthias Heinkenschloss, Danny C. Sorensen

Department of Computational and Applied Mathematics, MS-134, Rice University, 6100 Main Street, Houston, Texas 77005-1892, USA. e-mail: kleinsun/heinken/sorensen@ rice.edu

Roland Glowinski

University of Houston, Department of Mathematics, 651 P. G. Hoffman Hall, Houston, Texas

77204-3008, USA. e-mail: roland@math.uh.edu 
$[9,14]$, there are no bounds available for the error between the input-to-output map of the original system and that of the reduced order model. Proper Orthogonal Decomposition (POD) is often used for model reduction of nonlinear systems. Error bounds are available for the error between the so-called snapshots and the reduced order model, see, e.g., [11, 13], but no bounds for the error between the input-tooutput map of original system and that of the reduced order model, unless the socalled snapshot set reflects all possible inputs.

Our approach uses domain decomposition techniques to divide the problem into linear subproblems and small nonlinear subproblems. Balanced truncation is applied only to the linear subproblems with inputs and outputs determined by the original inand outputs as well as the interface conditions between the subproblems. We expect that this combination of domain decomposition and balanced truncation leads to a substantial reduction of the original problem if the nonlinearities are localized, i.e., the nonlinear subproblems are small relative to the other subdomains, and if the interfaces between the subproblems are relatively small.

To keep our presentation brief, we consider a model problem which couples the 1D Burgers equation to two heat equations. This is motivated by problems in which one is primarily interested in a nonlinear PDE which is posed on a subdomain and which is coupled to linear PDEs on surrounding, larger subdomains. The linear PDE solution on the surrounding subdomains needs to be computed accurately enough to provide acceptable boundary conditions for the nonlinear problem on the 'inner' subdomain. Such situations arise, e.g., in regional air quality models.

Our work is also related to [4], which is an example paper which discusses the coupling of linear and nonlinear PDEs, but no dimension reduction is applied. Domain decomposition and POD model reduction for flow problems with moving shocks are discussed in [15]. POD model reduction is applied on the subdomains away from the shock. The paper [18] discusses a different model reduction technique for second order dynamical systems with localized nonlinearities. The papers $[2,5]$ and [20] discuss different model reduction and substructuring techniques for second order dynamical systems and model reduction of interconnect systems respectively.

\section{The Model Problem}

Let $\bar{\Omega}=\bigcup_{k=1}^{3} \bar{\Omega}_{k}$, where $\Omega_{1}=(-10,-1), \Omega_{2}=(-1,1)$ and $\Omega_{3}=(1,10)$ and let $T>0$ be given. Our model problem is given by

$$
\begin{array}{rlrl}
\rho_{k} \frac{\partial y_{k}}{\partial t}(x, t)-\mu_{k} \frac{\partial^{2} y_{k}}{\partial x^{2}}(x, t) & =S_{k}(x, t), & (x, t) \in \Omega_{k} \times(0, T), \\
y_{k}(x, 0)=y_{k 0}(x), & x \in \Omega_{k}, k=1,3, \\
\frac{\partial y_{1}}{\partial x}(-10, t)=0, & \frac{\partial y_{3}}{\partial x}(10, t)=0 & t \in(0, T),
\end{array}
$$




$$
\begin{aligned}
\rho_{2} \frac{\partial y_{2}}{\partial t}(x, t)-\mu_{2} \frac{\partial^{2} y_{2}}{\partial x^{2}}+y_{2} \frac{\partial y_{2}}{\partial x}(x, t) & =0, & (x, t) \in \Omega_{2} \times(0, T), \\
y_{2}(x, 0) & =y_{20}(x), & x \in \Omega_{2},
\end{aligned}
$$

with the following interface conditions

$$
\begin{aligned}
& y_{1}(-1, t)=y_{2}(-1, t), \quad y_{2}(1, t)=y_{3}(1, t), \quad t \in(0, T), \\
& \mu_{1} \frac{\partial y_{1}}{\partial x}(-1, t)=\mu_{2} \frac{\partial y_{2}}{\partial x}(-1, t), \quad \mu_{2} \frac{\partial y_{2}}{\partial x}(1, t)=\mu_{3} \frac{\partial y_{3}}{\partial x}(1, t), \quad t \in(0, T) .
\end{aligned}
$$

We assume that the forcing functions $S_{1}, S_{3}$ are given by

$$
S_{k}=\sum_{i=1}^{n_{s}} b_{i k}(x) u_{i k}(t), \quad k=1,3 .
$$

To obtain the weak form of (1) and (2), we multiply the differential equations (1a, d) by test functions $v_{i} \in H^{1}\left(\Omega_{i}\right), i=1,2,3$, respectively, integrate over $\Omega_{i}$, and apply integration by parts. Using the boundary conditions $(1 \mathrm{c}, \mathrm{h})$ this leads to

$$
\begin{gathered}
\rho_{k} \frac{d}{d t} \int_{\Omega_{k}} y_{k} v_{k} d x+\mu_{k} \int_{\Omega_{k}} \frac{\partial y_{k}}{\partial x} \frac{\partial v_{k}}{\partial x} d x-\left.\mu_{k} \frac{\partial y_{k}}{\partial x} v_{k}\right|_{\partial \Omega_{k}}=\int_{\Omega_{k}} S_{k} v_{k} d x, k=1,3, \\
\rho_{2} \frac{d}{d t} \int_{\Omega_{2}} y_{2} v_{2} d x+\mu_{2} \int_{\Omega_{2}} \frac{\partial y_{2}}{\partial x} \frac{\partial v_{2}}{\partial x} d x+\int_{\Omega_{2}} \frac{\partial y_{2}}{\partial x} y_{2} v_{2} d x-\left.\mu_{2} \frac{\partial y_{2}}{\partial x} v_{2}\right|_{-1} ^{1}=0 .
\end{gathered}
$$

If $v_{k} \in H^{1}\left(\Omega_{k}\right), k=1,3$, satisfy $v_{1}(-1)=1, v_{3}(1)=1$, then (1c), (4a) imply

$$
\begin{aligned}
\mu_{1} \frac{\partial y_{1}(-1)}{\partial x} & =-\int_{\Omega_{1}} S_{1} v_{1} d x+\rho_{1} \frac{\partial}{\partial t} \int_{\Omega_{1}} y_{1} v_{1} d x+\mu_{1} \int_{\Omega_{1}} \frac{\partial y_{1}}{\partial x} \frac{\partial v_{1}}{\partial x} d x, \\
\mu_{3} \frac{\partial y_{3}(1)}{\partial x} & =\int_{\Omega_{3}} S_{3} v_{3} d x-\rho_{3} \frac{\partial}{\partial t} \int_{\Omega_{3}} y_{3} v_{3} d x-\mu_{3} \int_{\Omega_{3}} \frac{\partial y_{3}}{\partial x} \frac{\partial v_{3}}{\partial x} d x .
\end{aligned}
$$

If $v_{2} \in H^{1}\left(\Omega_{2}\right)$ satisfies $v_{2}(-1)=1$ and $v_{2}(1)=0$, then (4b) implies

$$
\mu_{2} \frac{\partial y_{2}(-1)}{\partial x}=-\rho_{2} \frac{\partial}{\partial t} \int_{\Omega_{2}} y_{2} v_{2} d x-\mu_{2} \int_{\Omega_{2}} \frac{\partial y_{2}}{\partial x} \frac{\partial v_{2}}{\partial x} d x-\int_{\Omega_{2}} \frac{\partial y_{2}}{\partial x} y_{2} v_{2} d x .
$$

Finally, if $v_{2} \in H^{1}\left(\Omega_{2}\right)$ satisfies $v_{2}(-1)=0$ and $v_{2}(1)=1$, then (4b) implies

$$
\mu_{2} \frac{\partial y_{2}(1)}{\partial x}=\rho_{2} \frac{\partial}{\partial t} \int_{\Omega_{2}} y_{2} v_{2} d x+\mu_{2} \int_{\Omega_{2}} \frac{\partial y_{2}}{\partial x} \frac{\partial v_{2}}{\partial x} d x+\int_{\Omega_{2}} \frac{\partial y_{2}}{\partial x} y_{2} v_{2} d x .
$$

The identities (5) are used to enforce the interface conditions (2).

We discretize the differential equations in space using piecewise linear functions. We subdivide $\Omega_{j}, j=1,2,3$, into subintervals. Let $x_{i}$ denote the subinterval endpoints and let $v_{i}$ be the piecewise linear basis function with $v_{i}\left(x_{i}\right)=1$ and $v_{i}\left(x_{j}\right)=0$ or all $j \neq i$. We define the following index sets 


$$
\begin{aligned}
& I_{1}^{I}=\left\{i: x_{i} \in[-10,-1)\right\}, \quad I_{2}^{I}=\left\{i: x_{i} \in(-1,1)\right\}, \quad I_{3}^{I}=\left\{i: x_{i} \in(1,10]\right\}, \\
& I_{12}^{\Gamma}=\left\{i: x_{i}=-1\right\}, \quad I_{23}^{\Gamma}=\left\{i: x_{i}=1\right\} \text {. }
\end{aligned}
$$

Given $y_{i}$ for $i \in I_{12}^{\Gamma} \cup I_{23}^{\Gamma}$, we compute functions

$$
\begin{aligned}
& y_{k}(t, x)=\sum_{i \in I_{k}^{I}} y_{i}(t) v_{i}(x)+\sum_{i \in I^{\Gamma}} y_{i}(t) v_{i}(x), \quad k=1,3, \\
& y_{2}(t, x)=\sum_{i \in I_{1}^{I}} y_{i}(t) v_{i}(x)+\sum_{i \in I_{12}^{\Gamma}} y_{i}(t) v_{i}(x)+\sum_{i \in I_{23}^{\Gamma}} y_{i}(t) v_{i}(x),
\end{aligned}
$$

where in (6a) we use $I^{\Gamma}=I_{12}^{\Gamma}$ if $k=1$ and $I^{\Gamma}=I_{23}^{\Gamma}$ if $k=3$, as solutions of

$$
\begin{gathered}
\rho_{k} \frac{d}{d t} \int_{\Omega_{k}} y_{k} v_{i} d x+\mu_{k} \int_{\Omega_{k}} \frac{\partial}{\partial x} y_{k} \frac{d}{d x} v_{i} d x=\int_{\Omega_{k}} S_{k} v_{i} d x, i \in I_{k}^{I}, \quad k=1,3, \\
\rho_{2} \frac{d}{d t} \int_{\Omega_{2}} y_{2} v_{i} d x+\mu_{2} \int_{\Omega_{2}} \frac{\partial}{\partial x} y_{2} \frac{d}{d x} v_{i} d x+\int_{\Omega_{2}} \frac{\partial}{\partial x} y_{2} y_{2} v_{i} d x=0, i \in I_{2}^{I} .
\end{gathered}
$$

If we set $\mathbf{y}_{k}^{I}=\left(y_{i}\right)_{i \in I_{k}^{I}}, k=1,2,3, \mathbf{y}_{j k}^{\Gamma}=\left(y_{i}\right)_{i \in I_{j k}^{\Gamma}}, j k \in\{12,23\}, \mathbf{y}^{\Gamma}=\left(\mathbf{y}_{12}^{\Gamma}, \mathbf{y}_{23}^{\Gamma}\right)^{T}$, and $\mathbf{u}_{k}=\left(u_{i}\right)_{i=1, \ldots, n_{s}}, k=1,3$ (cf. (3)), the previous identities can be written as

$$
\begin{aligned}
& \mathbf{M}_{1}^{I I} \frac{d}{d t} \mathbf{y}_{1}^{I}+\mathbf{A}_{1}^{I I} \mathbf{y}_{1}^{I}+\mathbf{M}_{1}^{I \Gamma} \frac{d}{d t} \mathbf{y}_{12}^{\Gamma}+\mathbf{A}_{1}^{I \Gamma} \mathbf{y}_{12}^{\Gamma}=\mathbf{B}_{1}^{I} \mathbf{u}_{1}, \\
& \mathbf{M}_{2}^{I I} \frac{d}{d t} \mathbf{y}_{2}^{I}+\mathbf{A}_{2}^{I I} \mathbf{y}_{2}^{I}+\mathbf{M}_{2}^{I \Gamma} \frac{d}{d t} \mathbf{y}^{\Gamma}+\mathbf{A}_{2}^{I \Gamma} \mathbf{y}^{\Gamma}+\mathbf{N}^{I}\left(\mathbf{y}_{2}^{I}, \mathbf{y}^{\Gamma}\right)=\mathbf{0}, \\
& \mathbf{M}_{3}^{I I} \frac{d}{d t} \mathbf{y}_{3}^{I}+\mathbf{A}_{3}^{I I} \mathbf{y}_{3}^{I}+\mathbf{M}_{3}^{I \Gamma} \frac{d}{d t} \mathbf{y}_{23}^{\Gamma}+\mathbf{A}_{3}^{I \Gamma} \mathbf{y}_{23}^{\Gamma}=\mathbf{B}_{3}^{I} \mathbf{u}_{3} .
\end{aligned}
$$

By construction, the functions $y_{j}, j=1,2,3$, in (6) satisfy (2a). To enforce (2b) we insert the identities (5), (6) into (2b). The resulting conditions can be written as

$$
\begin{aligned}
& \mathbf{M}_{1}^{\Gamma I} \frac{d}{d t} \mathbf{y}_{1}^{I}+\mathbf{A}_{1}^{\Gamma I} \mathbf{y}_{1}^{I}+\left(\mathbf{M}_{1}^{\Gamma \Gamma}+\mathbf{M}_{12}^{\Gamma \Gamma}\right) \frac{d}{d t} \mathbf{y}_{12}^{\Gamma}+\left(\mathbf{A}_{1}^{\Gamma \Gamma}+\mathbf{A}_{12}^{\Gamma \Gamma}\right) \mathbf{y}_{12}^{\Gamma} \\
&+ \mathbf{M}_{12}^{\Gamma I} \frac{d}{d t} \mathbf{y}_{2}^{I}+\mathbf{A}_{12}^{\Gamma I} \mathbf{y}_{2}^{I}+\mathbf{N}_{12}^{\Gamma}\left(\mathbf{y}_{2}^{I}, \mathbf{y}_{12}^{\Gamma}\right)=\mathbf{B}_{1}^{\Gamma} \mathbf{u}_{1}, \\
& \mathbf{M}_{3}^{\Gamma I} \frac{d}{d t} \mathbf{y}_{3}^{I}+\mathbf{A}_{3}^{\Gamma I} \mathbf{y}_{3}^{I}+\left(\mathbf{M}_{3}^{\Gamma \Gamma}+\mathbf{M}_{23}^{\Gamma \Gamma}\right) \frac{d}{d t} \mathbf{y}_{23}^{\Gamma}+\left(\mathbf{A}_{3}^{\Gamma \Gamma}+\mathbf{A}_{23}^{\Gamma \Gamma}\right) \mathbf{y}_{23}^{\Gamma} \\
&+\mathbf{M}_{23}^{\Gamma I} \frac{d}{d t} \mathbf{y}_{2}^{I}+\mathbf{A}_{23}^{\Gamma I} \mathbf{y}_{2}^{I}+\mathbf{N}_{23}^{\Gamma}\left(\mathbf{y}_{2}^{I}, \mathbf{y}_{23}^{\Gamma}\right)=\mathbf{B}_{3}^{\Gamma} \mathbf{u}_{3} .
\end{aligned}
$$

To summarize, our discretization of (1) and (2) is given by (7) and (8).

As outputs we are interested in the solution of the PDE at the spatial locations $\xi_{1}=-5, \xi_{2}=0, \xi_{3}=5$. Thus the output equations are $y_{k}\left(t, \xi_{k}\right)=\sum_{i \in I_{k}^{I}} y_{i}(t) v_{i}\left(\xi_{k}\right)$, $k=1,2,3$, which can be written as

$$
\mathbf{z}_{k}^{I}(t)=\mathbf{C}_{j}^{I} \mathbf{y}_{k}^{I}(t), \quad \text { where } \mathbf{C}_{k}^{I} \in \mathbb{R}^{1 \times\left|I_{k}^{I}\right|}, \quad k=1,2,3 .
$$




\section{Balanced Truncation Model Reduction}

Given $\mathscr{E} \in \mathbb{R}^{n \times n}$ symmetric positive definite, $\mathscr{A} \in \mathbb{R}^{n \times n}, \mathscr{B} \in \mathbb{R}^{n \times m}, \mathscr{C} \in \mathbb{R}^{q \times n}$, and $\mathscr{D} \in \mathbb{R}^{q \times m}$, we consider linear time invariant systems in state space form

$$
\begin{aligned}
\mathscr{E} \frac{d}{d t} \mathbf{y}(t) & =\mathscr{A} \mathbf{y}(t)+\mathscr{B} \mathbf{u}(t), \quad t \in(0, T), & \mathbf{y}(0)=\mathbf{y}_{0} \\
\mathbf{z}(t) & =\mathscr{C} \mathbf{y}(t)+\mathscr{D} \mathbf{u}(t), \quad t \in(0, T) &
\end{aligned}
$$

Projection methods for model reduction generally produce $n \times r$ matrices $\mathscr{V}, \mathscr{W}$ with $r \ll n$ and with $\mathscr{W}^{T} \mathscr{E} \mathscr{V}=I_{r}$. One obtains a reduced form of equations (9) by setting $\mathbf{y}=\mathscr{V} \widehat{\mathbf{y}}$ and projecting (imposing a Galerkin condition) so that

$$
\mathscr{W}^{T}\left[\mathscr{E} \mathscr{V} \frac{d}{d t} \widehat{\mathbf{y}}(t)-\mathscr{A} \mathscr{V} \widehat{\mathbf{y}}(t)-\mathscr{B} \mathbf{u}(t)\right]=0, \quad t \in(0, T)
$$

This leads to a reduced system of order $r$ with matrices $\widehat{\mathscr{E}}=\mathscr{W}^{T} \mathscr{E} \mathscr{V}=I_{k}, \widehat{\mathscr{A}}=$ $\mathscr{W}^{T} \mathscr{A} \mathscr{V}, \widehat{\mathscr{B}}=\mathscr{W}^{T} \mathscr{B}, \widehat{\mathscr{C}}=\mathscr{C} \mathscr{V}$, and $\widehat{\mathscr{D}}=\mathscr{D}$.

Balanced reduction is a particular techniqe for constructing the projecting matrices $\mathscr{V}$ and $\mathscr{W}$, see, e.g., $[1,16]$. One first solves the controllability and the observability Lyapunov equation $\mathscr{A} \mathscr{P} \mathscr{E}+\mathscr{E} \mathscr{P} \mathscr{A}^{T}+\mathscr{B} \mathscr{B}^{T}=0$ and $\mathscr{A}^{T} \mathscr{Q} \mathscr{E}+\mathscr{E} \mathscr{Q} \mathscr{A}+$ $\mathscr{C}^{T} \mathscr{C}^{T}=0$, respectively. Under the assumptions of stability, controllability and observability, the matrices $\mathscr{P}, \mathscr{Q}$ are both symmetric and positive definite. There exist methods to compute (approximations of) $\mathscr{P}=\mathbf{U} \mathbf{U}^{T}$ and $\mathscr{Q}=\mathbf{L} \mathbf{L}^{T}$ in factored form. In the large scale setting the factorization is typically a low rank approximation. See, e.g., $[8,17]$.

The balancing transformation is constructed by computing the singular value decomposition $\mathbf{U}^{T} \mathscr{E} \mathbf{L}=\mathbf{Z S Y} \mathbf{Y}^{T}$ and then setting $\mathscr{W}=\mathbf{U Z}_{r}, \mathscr{V}=\mathbf{L} \mathbf{Y}_{r}$, where $\mathbf{S}_{r}=$ $\operatorname{diag}\left(\sigma_{1}, \sigma_{2}, \ldots, \sigma_{r}\right)$ is the $r \times r$ submatrix of $\mathbf{S}=\mathbf{S}_{n}$. The singular values $\sigma_{j}$ are in decreasing order and $r$ is selected to be the smallest positive integer such that $\sigma_{r+1}<\tau \sigma_{1}$ where $\tau>0$ is a prespecified constant. The matrices $\mathbf{Z}_{r}, \mathbf{Y}_{r}$ consist of the corresponding leading $k$ columns of $\mathbf{Z}, \mathbf{Y}$.

It is well known [6] that $\widehat{\mathscr{A}}$ must be stable and that for any given input u we have

$$
\|\mathbf{z}-\widehat{\mathbf{z}}\|_{\mathscr{L}_{2}} \leq 2\|\mathbf{u}\|_{\mathscr{L}_{2}}\left(\sigma_{r+1}+\ldots+\sigma_{n}\right)
$$

where $\widehat{\mathbf{z}}$ is the output (response) of the reduced model. Model reduction techniques for infinite dimensional systems are reviewed in, e.g., [3].

We want to apply balanced truncation model to the linear subsystems 1 and 3 in (7) and (8). We need to identify the input-output relations for these subsystems in the context of the coupled system to ensure that balancing techniques applied to these subsystems leads to a reduced model for the coupled system with error bounds.

To identify the appropriate input-output relations, we focus on subsystem 1. Examination of (7a,b) and (8a) shows that $\mathbf{M}_{1}^{I \Gamma} \frac{d}{d t} \mathbf{y}_{12}^{\Gamma}, \mathbf{A}_{1}^{I \Gamma} \mathbf{y}_{12}^{\Gamma}$ and $\mathbf{B}_{1}^{I} \mathbf{u}_{1}$ are the inputs into system 1 and $\mathbf{C}_{1}^{\Gamma I} \mathbf{y}_{1}^{I}, \mathbf{M}_{1}^{\Gamma I} \frac{d}{d t} \mathbf{y}_{1}^{I}+\mathbf{A}_{1}^{\Gamma I} \mathbf{y}_{1}^{I}$ are the outputs. Hence, if 


$$
\mathbf{M}_{1}^{I \Gamma}=\mathbf{0} \quad \text { and } \quad \mathbf{M}_{1}^{\Gamma I}=\mathbf{0},
$$

then we need to apply model reduction to

$$
\begin{aligned}
\mathbf{M}_{1}^{I I} \frac{d}{d t} \mathbf{y}_{1}^{I} & =-\mathbf{A}_{1}^{I I} \mathbf{y}_{1}^{I}-\mathbf{A}_{1}^{I \Gamma} \mathbf{y}_{12}^{\Gamma}+\mathbf{B}_{1}^{I} \mathbf{u}_{1} \\
\mathbf{z}_{1}^{I} & =\mathbf{C}_{1}^{I} \mathbf{y}_{1}^{I}, \quad \mathbf{z}_{1}^{\Gamma}=\mathbf{A}_{1}^{\Gamma I} \mathbf{y}_{1}^{I} .
\end{aligned}
$$

The system (12) is exactly of the form (9) and we can apply balanced truncation model reduction to obtain

$$
\begin{aligned}
\widehat{\mathbf{M}}_{1}^{I I} \frac{d}{d t} \widehat{\mathbf{y}}_{1}^{I} & =-\widehat{\mathbf{A}}_{1}^{I} \widehat{\mathbf{y}}_{1}^{I}-\widehat{\mathbf{A}}_{1}^{I \Gamma} \mathbf{y}_{12}^{\Gamma}+\widehat{\mathbf{B}}_{1}^{I} \mathbf{u}_{1} \\
\widehat{\mathbf{z}}_{1}^{I} & =\widehat{\mathbf{C}}_{1}^{I} \widehat{\mathbf{y}}_{1}^{I}, \quad \widehat{\mathbf{z}}_{1}^{\Gamma}=\widehat{\mathbf{A}}_{1}^{I I} \widehat{\mathbf{y}}_{1}^{I} .
\end{aligned}
$$

Subsystem 3 can be reduced analogously. The reduced model for the coupled nonlinear system (7) and (8) is now obtained by replacing the subsystem matrices for subsystems 1 and 3 by their reduced matrices. Whether the balanced truncation error bound (10) can be used to derive an error bound between the original coupled problem (7) and (8) and its reduced model is under investigation.

In our finite element discretization we use mass lumping to obtain (11). However other discretizations, such as spectral elements or discontinuous Galerkin methods satisfy (11) directly, see [10, 12].

\section{Numerical Results}

We subdivide $\Omega_{j}$ into equidistant subintervals of length $h_{k}=1 / N_{k}, k=1,2,3$, and we use piecewise linear basis functions. The size of the system (7), (8) is $9\left(N_{1}+\right.$ $\left.N_{3}\right)+2 N_{2}+1$. The parameters in the PDE are $\rho_{k}=1, k=1,2,3$, and $\mu_{1}=0.05, \mu_{2}=$ $0.1, \mu_{1}=0.2$. For subsystem 1 and 3 we compute low-rank approximate solutions of the controllability and observability Lyapunov equations using the method described in [8]. We truncate such that $\sigma_{r+1}<\tau \sigma_{1}$, where $\tau=10^{-4}$.

The sizes of the full and of the reduced order models for various discretization parameters are shown in Table 1. The subsystems 1 and 3 reduce substantially and the size of the subsystem 2 limits the amount of reduction achieved overall. For example, for $N_{1}=N_{3}=20$ the subsystems 1 and 3 are each reduced in size from 180 to 11 . The size of the coupled system is reduced from $361+2 N_{2}$ to $23+2 N_{2}$.

Next, we compare the system output given forcing functions $S_{1}=u_{1}(t), S_{3}=$ $u_{3}(t)$ (cf., (3)) with $u_{1}(t)=\frac{1}{2} \sin (3 t)(1-0.8 t / T), u_{3}(t)=\sin (2 t)(0.3+0.7 t / T)$ on $(0, T)=(0,15)$. The full order model $(7),(8)$ and the corresponding reduced order model are solved using the modified $\theta$-scheme [7, 19] with (macro) time step $\Delta t=$ $T / 200$. Figure 1 shows the outputs, i.e., the approximate solution of the PDE at $\xi_{1}=$ $-5, \xi_{2}=0, \xi_{3}=5$. The left plot in Figure 2 shows the solution of the reduced order discretized PDE. The solution of the discretized PDE is visually indistinguishable 
Table 1 Dimension of the full and of the reduced order models for various discretization parameters $N_{1}, N_{2}, N_{3}$ and $\tau=10^{-4}$.

\begin{tabular}{llll}
\hline$N_{1}=N_{3}$ & $N_{2}$ & size of full order model & size of reduced order model \\
\hline 10 & 10 & 201 & 41 \\
20 & 20 & 401 & 63 \\
40 & 40 & 801 & 107 \\
20 & 10 & 381 & 43 \\
40 & 20 & 761 & 67 \\
\hline
\end{tabular}

Fig. 1 Outputs 1, 2, 3 of the full order system corresponding to the discretization $N_{1}=N_{2}=N_{3}=10$ are given by $*, \circ$ and $\square$, respecitively. Outputs 1,2, 3 of the reduced order system are given by dotted, dashed and solid lines, respectively.
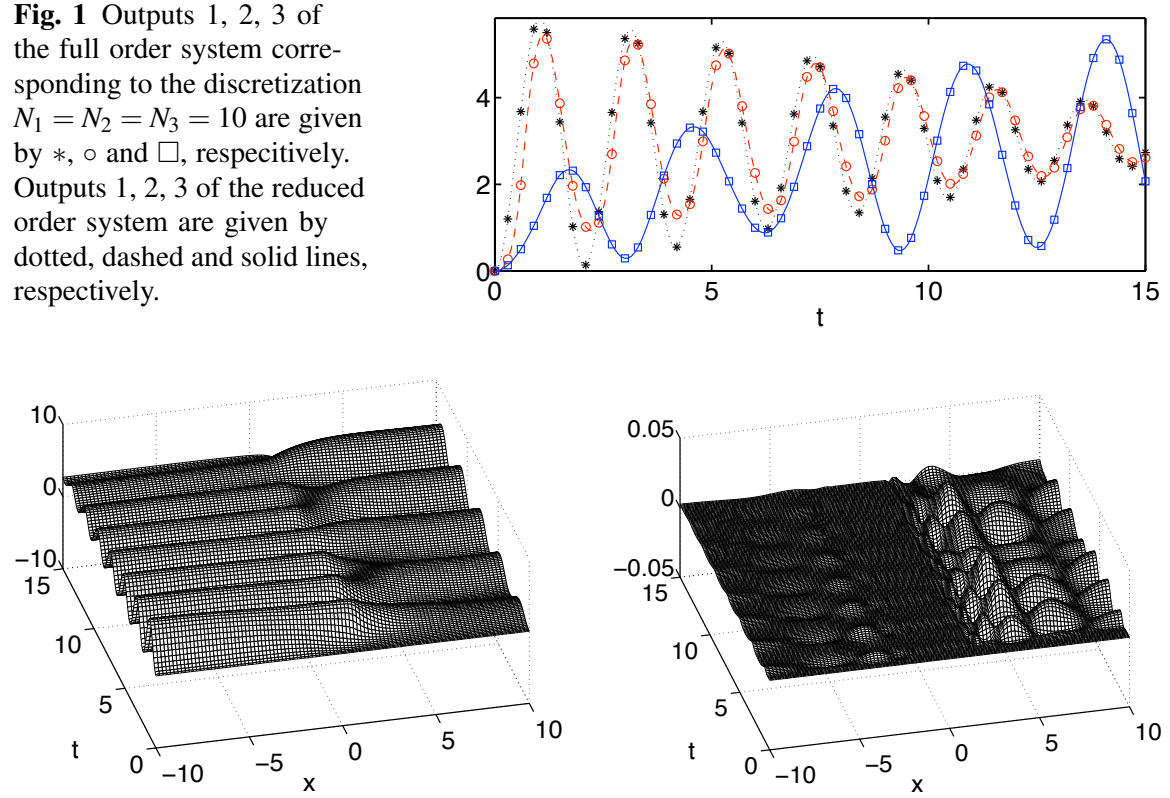

Fig. 2 Solution of the reduced order discretized PDE (left) and error between the solution of the discretized PDE and the reduced order system (right) for discretization $N_{1}=N_{2}=N_{3}=10$.

from the solution of the reduced order discretized PDE, as indicated by the size of the error shown in the right plot in Figure 2. The error is larger in the right subdomain because the PDE solution is positive and the advection term in (1d) advects the solution to the right.

Our numerical results indicate that the coupling of balanced truncation reduction for linear time variant subsystems with spatially localized nonlinear models leads to a coupled reduced order model with an error in the input-to-output map that is comparable to the error due to balanced truncation model reduction applied to the linear subsystems alone. The efficiency of the approach depends on the size of the interface and on the size of the localized nonlinearity. Investigations for higher dimensional problems are underway to explore the overall gains in efficiency. 
Acknowledgements This research was supported in part by NSF grant ACI-0121360 and AFOSR grant FA9550-06-1-0245.

\section{References}

1. Antoulas, A.C.: Approximation of Large-Scale Systems. SIAM, Philadelphia (2005)

2. Bai, Z., Liao, B.S.: Towards an optimal substructuring method for model reduction. In: J. Dongarra, K. Madsen, J. Wasniewski (eds.) Applied Parallel Computing, pp. 276-285. Springer Lecture Notes in Computer Science, Vol. 3732 (2006)

3. Curtain, R.F.: Model reduction for control design for distributed parameter systems. In: Research directions in distributed parameter systems (Raleigh, NC, 2000), Frontiers Appl. Math., vol. 27, pp. 95-121. SIAM, Philadelphia, PA (2003)

4. Discacciati, M., Quarteroni, A.: Convergence analysis of a subdomain iterative method for the finite element approximation of the coupling of Stokes and Darcy equations. Comput. Vis. Sci. 6(2-3), 93-103 (2004)

5. Givoli, D., Barbone, P.E., Patlashenko, I.: Which are the important modes of a subsystem? Internat. J. Numer. Methods Engrg. 59(12), 1657-1678 (2004)

6. Glover, K.: All optimal Hankel-norm approximations of linear multivariable systems and their $L^{\infty}$-error bounds. Internat. J. Control 39(6), 1115-1193 (1984)

7. Glowinski, R.: Finite element methods for incompressible viscous flow. Handbook of Numerical Analysis, Vol. IX. North-Holland, Amsterdam (2003)

8. Gugercin, S., Sorensen, D.C., Antoulas, A.C.: A modified low-rank Smith method for largescale Lyapunov equations. Numer. Algorithms 32(1), 27-55 (2003)

9. Hahn, J., Edgar, T.F.: An improved method for nonlinear model reduction using balancing of empirical Gramians. Computers and Chemical Engineering 26, 1379-1397 (2002)

10. Hesthaven, J.S., Warburton, T.: Nodal Discontinuous Galerkin Methods: Analysis, Algorithms, and Applications. Springer-Verlag, Berlin (2008)

11. Hinze, M., Volkwein, S.: Proper orthogonal decomposition surrogate models for nonlinear dynamical systems: Error estimates and suboptimal control. In: P. Benner, V. Mehrmann, D.C. Sorensen (eds.) Dimension Reduction of Large-Scale Systems, Lecture Notes in Computational Science and Engineering, Vol. 45, pp. 261-306. Springer-Verlag, Heidelberg (2005)

12. Karniadakis, G.E., Sherwin, S.J.: Spectral/ $h p$ Element Methods for Computational Fluid Dynamics, second edn. Oxford University Press (2005)

13. Kunisch, K., Volkwein, S.: Galerkin proper orthogonal decomposition methods for a general equation in fluid dynamics. SIAM J. Numer. Anal. 40(2), 492-515 (electronic) (2002)

14. Lall, S., Marsden, J.E., Glavaški, S.: A subspace approach to balanced truncation for model reduction of nonlinear control systems. Int. J. Robust Nonlinear Control 12(6), 519-535 (2002)

15. Lucia, D.J., King, P.I., Beran, P.S.: Reduced order modeling of a two-dimensional flow with moving shocks. Computer and Fluids 32, 917-938 (2003)

16. Moore, B.C.: Principal component analysis in linear systems: controllability, observability, and model reduction. IEEE Trans. Automat. Control 26(1), 17-32 (1981)

17. Penzl, T.: Algorithms for model reduction of large dynamical systems. Linear Algebra Appl. 415(2-3), 322-343 (2006)

18. Segalman, D.J.: Model reduction of systems with localized nonlinearities. Journal of Computational and Nonlinear Dynamics 2(3), 249-266 (2007)

19. Turek, S., Rivkind, L., Hron, J., Glowinski, R.: Numerical study of a modified time-stepping $\theta$-scheme for incompressible flow simulations. J. Sci. Comput. 28(2-3), 533-547 (2006)

20. Vandendorpe, A., Dooren, P.V.: On model reduction of interconnected systems. In: W. Schilders, H. van der Vorst (eds.) Model Order Reduction: Theory, Research Aspects and Applications, Mathematics in Industry, pp. 160-175. Springer Verlag, Heidelberg (2007) 